\title{
Text Anchor Based Metric Learning for Small-footprint Keyword Spotting
}

\author{
Li Wang ${ }^{1}$, Rongzhi Gu ${ }^{1}$, Nuo Chen ${ }^{1}$, Yuexian Zou ${ }^{1,2, *}$ \\ ${ }^{1}$ ADSPLAB, School of ECE, Peking University, Shenzhen, China \\ ${ }^{2}$ Peng Cheng Laboratory, Shenzhen, China \\ \{1901213145, 1701111335, nuochen, zouyx\}@pku.edu.cn
}

\begin{abstract}
Keyword Spotting (KWS) remains challenging to achieve the trade-off between small footprint and high accuracy. Recently proposed metric learning approaches improved the generalizability of models for the KWS task, and 1D-CNN based KWS models have achieved the state-of-the-arts (SOTA) in terms of model size. However, for metric learning, due to data limitations, the speech anchor is highly susceptible to the acoustic environment and speakers. Also, we note that the 1D-CNN models have limited capability to capture long-term temporal acoustic features. To address the above problems, we propose to utilize text anchors to improve the stability of anchors. Furthermore, a new type of model (LG-Net) is exquisitely designed to promote long-short term acoustic feature modeling based on 1D-CNN and self-attention. Experiments are conducted on Google Speech Commands Dataset version 1 (GSCDv1) and 2 (GSCDv2). The results demonstrate that the proposed text anchor based metric learning method shows consistent improvements over speech anchor on representative CNN-based models. Moreover, our LG-Net model achieves SOTA accuracy of $97.67 \%$ and $96.79 \%$ on two datasets, respectively. It is encouraged to see that our lighter LG-Net with only $74 \mathrm{k}$ parameters obtains $96.82 \%$ KWS accuracy on the GSCDv1 and $95.77 \%$ KWS accuracy on the GSCDv2.

Index Terms: keyword spotting, long-term information, metric learning, small-footprint, text anchor
\end{abstract}

\section{Introduction}

With speech technology development, speech assistants can help people solve affairs more efficiently, such as querying weather and controlling air conditioning. People increasingly enjoy the convenience of the hands-free experience. Keyword Spotting (KWS) is the beginning of the human-computer speech interaction, aims at distinguishing between each of the target keywords of interest and non-target sounds such as general speech (non-target words) and noises [1].

Recently, researchers have improved the generalization performance of models in terms of data enhancement [2], loss function [3,4], automatic gain control [5], negative sample mining [6], and metric learning $[1,7]$. In particular, the metric learning based on triplet loss $[1,7]$ has demonstrated excellent performance. The KWS models are trained using triplet consists of an anchor sample, a positive sample from the same class with the anchor, and a negative sample from a different class. The objective of the network training is to minimize the distance between the embeddings of the anchor and the positive sample while maximizing the distance between the embeddings of the anchor and the negative sample. It can be seen that the performance is much dependent on the choice of

*Corresponding author the anchor. However, due to data limitations, the speech anchor is highly susceptible to the acoustic environment and speakers, making the anchor embedding more variant during training. In addition, deviated speech anchors may make the KWS models suffering from a local optimality issue, which leads to the degradation of the performance.

On the other hand, Deep neural networks (DNNs) have recently proven to yield efficient small-footprint solutions for KWS $[8,9,10,11,12,13,14,15,16]$. In particular, more advanced architectures, such as Convolutional Neural Networks (CNNs), have been applied to solve KWS problems under limited memory footprint as well as computational resource scenarios, showing excellent accuracy. Most CNN-based KWS models receive features, such as Mel-Frequency Cepstral Coefficient (MFCC), as a 2D input. However, such 2D CNN-based KWS models struggle with capturing the dependency between low and high frequencies with the relatively shallow network. To address this problem, $[13,15]$ utilize temporal convolution to capture low and high-frequency features in a shallow network, which achieves the best KWS performance. Despite their success, due to local region perception and weight sharing characteristics of CNNs, the long-term temporal information may not be considered. Figure 1a shows the feature maps produced by a representative 1D-CNN-based KWS model, TC-ResNet141.5 [13]. It is noted that the saliency regions are distributed sparsely, which implies that 1D-CNN focuses on the short-term temporal information. In fact, it is crucial to capture the longterm temporal information for KWS, considering that the characteristics of keywords are usually different on the time scale.

In this study, we aim at boosting the performance of the small-footprint KWS model. To improve the stability of the anchor, we replace the speech anchor with the text anchor and propose a text anchor based metric learning method for KWS. Specifically, target keywords and non-target words are encoded in the text anchors by pretraining models (like BERT [17]). Moreover, the local-global network (LG-Net) is designed to model local and global information. Specifically, the selfattention layer [18] is stacked to the 1D temporal convolution layer to capture the long-term and short-term temporal information. Experimental results demonstrate that the proposed text anchor based metric learning method shows consistent improvements over speech anchor on representative CNN-based models. Besides, our LG-Net model achieves SOTA accuracy of $97.67 \%$ and $96.79 \%$ on Google Speech Commands Dataset version 1 (GSCDv1) and version 2 (GSCDv2), respectively.

\section{Proposed Method}

\subsection{Data Process}

The raw audio is decomposed into a sequence of frames where the window length is $25 \mathrm{~ms}$ and the stride is $10 \mathrm{~ms}$ for feature extraction. We use 40 Mel-Frequency Cepstral Coeffi- 


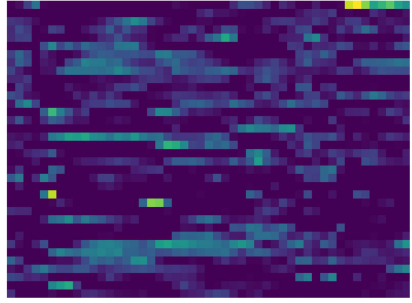

Frame

(a) Output of TC-ResNet14-1.5 $2^{\text {nd }}$ Block

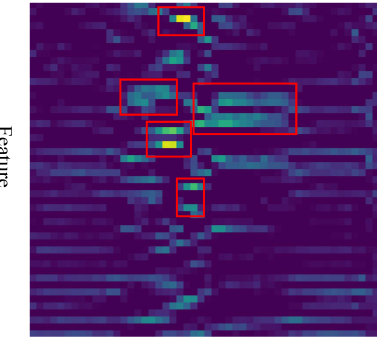

Frame

(b) 1D-CNN output of LG-Net6 $2^{\text {nd }}$ Block

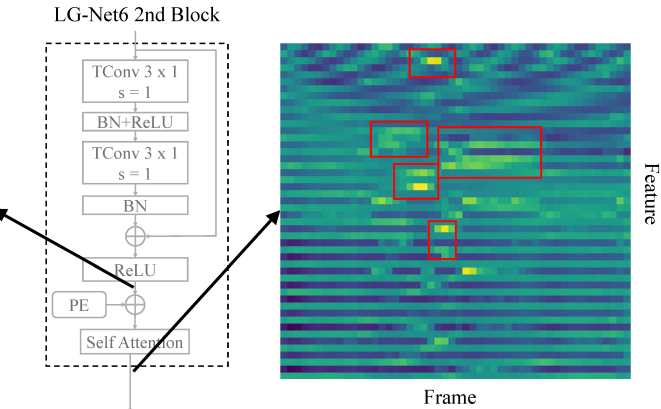

(c) Output of LG-Net6 $2^{\text {nd }}$ Block

Figure 1: Feature maps produced by the TC-ResNet14-1.5 and proposed model LG-Net using a benchmark image, saliency for each feature is represented by brightness. (a) and (b) shows that the feature maps outputs by $1 D$-CNN are sparse, representing that $1 D$-CNN focuses on short-term temporal information. (c) shows the self-attention layer modeling the long-term information while preserving the short-term temporal information captured by the $1 D-C N N$.

cient (MFCC) features for each frame and stack them over the time-axis, denote as $M \in \mathbb{R}^{T \times F}$ where $F$ represents the dimension of the MFCC feature, and $T$ denotes the number of frames. The text anchor is extracted by BERT-base [17] denoted as $V \in \mathbb{R}^{D}$, where $D$ represents the dimension of the text anchor (i.e. $D=768$ ).

\subsection{Text anchor based metric learning method}

As mentioned in Section 1, the susceptibility of the speech anchor leads to the poor performance of the model. Therefore, we propose a text anchor based metric learning method for KWS, illustrated in Figure 2b. The input triplet is denoted as $\left(M^{+}\right.$, $M^{-}, V$ ), where $M^{+}$and $M^{-}$are input MFCCs from different classes, and $V$ as the input text vector corresponding to $\mathrm{M}^{+}$. The speech embeddings $E_{\mathrm{S}}^{M^{+}} \in \mathbb{R}^{D^{\prime}}$ and $E_{\mathrm{S}}^{M^{-}} \in \mathbb{R}^{D^{\prime}}$ are extracted from $M^{+}$and $M^{-}$by the speech embedding extraction module $f_{\mathrm{S}}$, respectively, where $D^{\prime}$ represents the dimension of the speech embedding (i.e. $D^{\prime}=128$ ). In parallel, $V$ is mapped as a text embedding $E_{\mathrm{T}} \in \mathbb{R}^{D^{\prime}}$. The triplet loss is employed to decreases the distance between the $E_{\mathrm{T}}$ and $E_{\mathrm{S}}^{M^{+}}$ and increases the distance between $E_{\mathrm{T}}$ and $E_{\mathrm{S}}^{M^{-}}$. For a single sample, the triplet loss is thus

$$
L_{t r i}=\max \left(d\left(E_{\mathrm{S}}^{M^{+}}, E_{\mathrm{T}}\right)-d\left(E_{\mathrm{S}}^{M^{-}}, E_{\mathrm{T}}\right)+\alpha, 0\right)
$$

where $d(x, y)=\|x-y\|_{p}$ is the pairwise-distance between $\mathrm{x}$ and $\mathrm{y}$ (i.e. $p=2$ ); $\alpha$ is a constant margin (i.e. $\alpha=1$ ).

Note that the phone embeddings extracted by the text encoder in [7] is fundamentally different from the text anchor proposed in this paper. In [7], a pronunciation dictionary is used to map words to their sequence of phonemes. Therefore, the phone embeddings do not contain the information about other words, although it is not influenced by factors such as acoustic environment and speaker. The text anchor in this paper is extracted from the BERT [17] which is trained on the large-scale text corpus, it contains information about other words.

\subsection{Local-global Neural Network}

As mentioned in Section 1, for KWS, long-term temporal information is crucial because the characteristics of keywords are usually different on the time scale. In this work, we utilize the

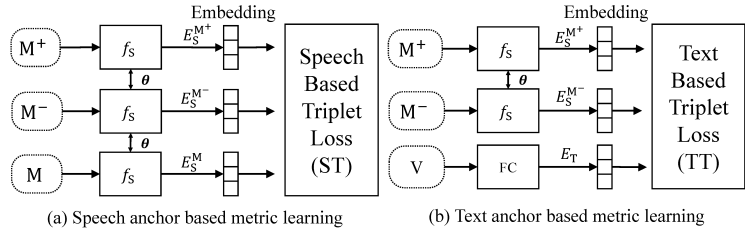

Figure 2: (a) Speech anchor based metric learning and (b) proposed text anchor based metric learning. $M^{+}$and $M^{-}$are input MFCCs from different classes, $M$ is input MFCC from the same class of $\mathrm{M}^{+}$, and $V$ as the input text vector encoded by BERT [17] corresponding $M^{+} . E_{\mathrm{S}}$ represents the speech embedding output from the speech embedding extraction module $f_{\mathrm{S}} . E_{\mathrm{T}}$ represents the text embedding.

self-attention [18] layer to capture long-term temporal information. The temporal convolution with residual structure [13] is adopt in this paper, which is one of the most widely used 1DCNN architectures for KWS. To reduce the number of parameters, $3 \times 1$ kernels are utilized instead of $9 \times 1$ kernels. As Figure 3 shows, and the self-attention layer is stacked to the 1DCNN layers, denoted as LG-Block. Following the design of the previous study [13], the identity shortcuts can be directly used when the input and output have matching dimensions (Figure $3 a)$; otherwise, we use an extra $1 \times 1$ convolution with a stride to match the dimensions (Figure 3b). Local-global neural network (LG-Net) can be easily constructed by stacking LG-Blocks. We select LG-Net6 (Figure 3c), which has 6 LG-Blocks as our base model. To establish a small-footprint model, we compress the LG-Net6 by taking out three LG-Blocks and reducing the number of channels, named LG-Net3.

Considering the variation in feature maps due to speaking style, speed of speech, we use an averaging pooling strategy on the time dimension. The output of the average pooling layer is given to a fully connected layer to generate speech embedding $E_{\mathrm{S}} \in \mathbb{R}^{D^{\prime}}$.

$$
E_{\mathrm{S}}=f_{\mathrm{S}}\left(M ; \theta_{\mathrm{S}}\right)
$$

where $\theta_{\mathrm{S}}$ denote the parameters of the $f_{\mathrm{S}}$.

Finally, the predicted score, $\hat{y} \in \mathbb{R}^{C}$ where $C$ denote the 


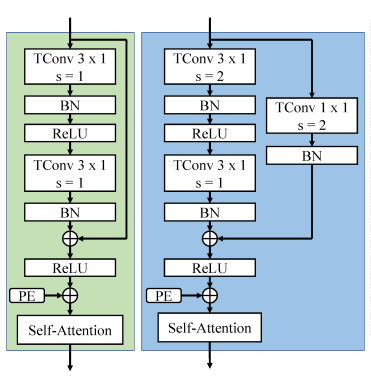

(a) LG-Block (s=1) (b) LG-Block ( $\mathrm{s}=2$ )

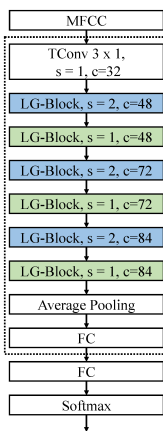

(c) LG-Net6

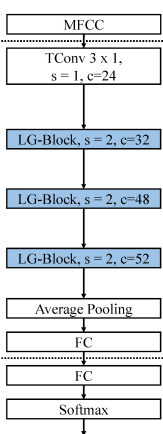

(d) LG-Net3
Figure 3: The local-global block (LG-Block) when (a) stride = 1 and (b) stride $=2$ and two implementations of local-global neural network (LG-Net) when (c) LG-Net6 and (d) LG-Net3. TConv means the temporal convolution layer, " $s$ " and " $c$ " indicates stride and channel of TConv, respectively. PE means the position encoding [18]. BN and FC denote batch normalization and fully connected layer. The part inside the dotted line is regarded as the speech embedding extraction module.

number of class, is obtained from $E_{\mathrm{S}}$

$$
\hat{y}=\sigma\left(f_{\mathrm{FC}}\left(E_{\mathrm{S}} ; \theta_{\mathrm{FC}}\right)\right)
$$

where $\sigma$ is the sigmoid function; $\theta_{\mathrm{FC}}$ denotes the parameters of the fully connected layer $f_{\mathrm{FC}}$. KWS as a multi-classification problem, the cross entropy (CE) loss is adopted to optimize the model, for the given ground truth $y \in \mathbb{R}^{C}$ (where $y^{(i)}=\{0,1\}$ denotes whether label $i$ appears or not), the loss $L$ is calculated using binary cross-entropy:

$$
L_{C E}=\sum_{i=1}^{C} y^{(i)} \log \left(\hat{y}^{(i)}\right)+\left(1-y^{(i)}\right) \log \left(1-\hat{y}^{(i)}\right)
$$

In this work, the networks are optimized by the loss $L$, which is calculated by weighted sum of $L_{\mathrm{tri}}$ and $L_{\mathrm{CE}}$

$$
L=\beta L_{\mathrm{tri}}+(1-\beta) L_{\mathrm{CE}}
$$

where $\beta$ is the balanced weight between $L_{\text {tri }}$ and $L_{\mathrm{CE}}$.

Except for the cases of target keywords and non-target words, a "silence" class is considered to indicate the case when no speech is detected [19]. However, in this work, the text vector of "silence" does not match the definition in KWS, so the model cannot be trained directly using the text vector of "silence". To address the problem, we first train the network without the "silence" sample and then finetune the two FC layers on the full dataset with only CE loss.

\section{Experiments}

\subsection{Datasets}

The proposed models are trained and evaluated with Google Speech Commands Dataset version $1\left(\mathrm{GSCDv}^{1}{ }^{1}\right)$ and version 2 (GSCDv2 ${ }^{2}$ ) [19]. Following the implementation of Google [19], we seek to discriminate among 12 classes for GSCDv1: "yes", "no", “up”, “down”, "left", “right”, “on”,

\footnotetext{
${ }^{1} \mathrm{http}: / /$ download.tensorflow.org/data/speech_commands_v0.01.tar.gz

${ }^{2} \mathrm{http} / / /$ download.tensorflow.org/data/speech_commands_v0.02.tar.gz
}

Table 1: The number of samples in training, validation and test set of google speech commands dataset version 1 and version 2.

\begin{tabular}{|l|c|c|c|c|}
\hline Dataset & Train & Valid & Test & Total \\
\hline GSCDv1 & 55287 & 7218 & 7242 & 69747 \\
GSCDv2 & 89043 & 10401 & 11413 & 110857 \\
\hline
\end{tabular}

"off", "stop", "go", unknown, or silence, and 16 classes for GSCDv2 which add 4 keywords of GSCDv1: "backward", "forward", "follow", and "learn". In order to generate background noise, we randomly sample and crop background noises provided in the dataset. For a fair comparison, in our test set, the "silence" class test samples are taken from open source speech commands dataset test set version $2^{3}$ [19], and test samples of other classes are written in the officially released testing.list ${ }^{12}$. Table 1 shows the number of samples in training, validation, and test sets. Note that to make it easy for other researchers to reproduce the model and results in this paper, we do not use any data augmentation methods.

\subsection{Implementation Details}

Our implementation was done with Pytorch [20] deep learning toolkit.

Training. All the models are trained with a mini-batch of 256 samples using stochastic gradient descent with weight decay of 0.001 and momentum of 0.9. For formula (5), the $L_{\text {tri }}$ and $L_{\mathrm{CE}}$ weights to be the same, i.e. $\beta=0.5, L=$ $0.5 L_{\mathrm{CE}}+0.5 L_{\mathrm{tri}}$. The initial learning rate is set to be 0.01 and decayed by a factor of 3 when the validation accuracy does not increase for 3 epochs. The training is terminated when validation accuracy does not increase for 10 epochs. For the finetuning stage mentioned in Section 2.3, the training setup is the same as the text anchor based metric learning method, except that $\beta$ is set as 0 .

Evaluation. The primary metric in our experiments is classification accuracy, the proportion of correct decisions out of the total number of samples. We also report the number of parameters and the false reject rate (FRR) at $0.5 \%$ false alarm rate (FAR). We train each model 10 times and report its average performance.

\subsection{Baselines}

To demonstrate the consistent performance improvement of our proposed text anchor based metric learning method, we reproduce CnnTradFpool3 [9], CnnOneFstride4 [9], TDNNSWSA [14], TC-ResNet8 [13], and TC-ResNet141.5 [13], which are representative CNN-based KWS models.

We use three loss functions for training and comparison. First, the CE loss, which is the most widely used loss function in the KWS task. Second, the triplet loss based on speech anchor $(S T)$ is used to evaluate the performance gains from the triplet loss. As shown in Figure 2a, the input triplet is denoted as $\left(\mathrm{M}^{+}\right.$, $M^{-}, M$ ), where $M$ is input MFCC randomly sampled from the training set with the same class of $M^{+}$. Third, the triplet loss based on text anchor (TT). As mentioned in Section 2.3, we employ CE loss accompanied by the ST and the TT, denote as $C E+S T$ and $C E+T T$, respectively. Also, to evaluate the performance improvement brought by our proposed LG-Net model, we selected several CNN-based KWS models trained with CE loss functions for direct comparison $[10,15,16]$.

\footnotetext{
${ }^{3}$ http://download.tensorflow.org/data/speech_commands_test_set_v0.02.tar.gz
} 
Table 2: Comparison of the proposed method and the baselines

\begin{tabular}{|c|c|c|c|c|}
\hline Model & Loss & Params & Acc.(v1) & Acc.(v2) \\
\hline \multirow{3}{*}{$\begin{array}{l}\text { CnnTradFpool3 [9] } \\
\text { (Sainath et al. 2015) }\end{array}$} & $\mathrm{CE}$ & & $93.53 \%$ & $90.15 \%$ \\
\hline & $\mathrm{CE}+\mathrm{ST}$ & $139 \mathrm{M}$ & $93.72 \%$ & $90.19 \%$ \\
\hline & $\mathrm{CE}+\mathrm{TT}$ & & $94.35 \%$ & $91.45 \%$ \\
\hline \multirow{3}{*}{$\begin{array}{l}\text { CnnOneFstride4 [9] } \\
\text { (Sainath } \text { et al. 2015) }\end{array}$} & $\mathrm{CE}$ & & $91.50 \%$ & $89.16 \%$ \\
\hline & $\mathrm{CE}+\mathrm{ST}$ & $3.84 \mathrm{M}$ & $91.68 \%$ & $90.03 \%$ \\
\hline & $\mathrm{CE}+\mathrm{TT}$ & & $92.21 \%$ & $90.38 \%$ \\
\hline \multirow{3}{*}{$\begin{array}{l}\text { TDNNSWSA [14] } \\
\text { (Bai et al. 2019) }\end{array}$} & $\mathrm{CE}$ & & $94.19 \%$ & $93.11 \%$ \\
\hline & $\mathrm{CE}+\mathrm{ST}$ & $20 \mathrm{~K}$ & $95.32 \%$ & $93.77 \%$ \\
\hline & $\mathrm{CE}+\mathrm{TT}$ & & $95.47 \%$ & $94.15 \%$ \\
\hline \multirow{3}{*}{$\begin{array}{l}\text { TC-ResNet8 [13] } \\
\text { (Choi et al. 2019) }\end{array}$} & $\mathrm{CE}$ & & $95.71 \%$ & $95.22 \%$ \\
\hline & $\mathrm{CE}+\mathrm{ST}$ & $72 \mathrm{~K}$ & $96.12 \%$ & $95.56 \%$ \\
\hline & $\mathrm{CE}+\mathrm{TT}$ & & $96.58 \%$ & $95.59 \%$ \\
\hline \multirow{3}{*}{$\begin{array}{l}\text { TC-ResNet14-1.5 [13] } \\
\text { (Choi } \text { et al. 2019) }\end{array}$} & $\mathrm{CE}$ & & $96.78 \%$ & $95.96 \%$ \\
\hline & $\mathrm{CE}+\mathrm{ST}$ & $313 \mathrm{~K}$ & $96.81 \%$ & $96.20 \%$ \\
\hline & $\mathrm{CE}+\mathrm{TT}$ & & $96.89 \%$ & $96.27 \%$ \\
\hline \multirow{4}{*}{$\begin{array}{l}\text { Resis* [10] } \\
\text { (Tang et al. 2018) } \\
\text { TENet12* }[15] \\
\text { (Li et al. 2020) } \\
\text { NAS2* [16] } \\
\text { (Mo et al. 2020) }\end{array}$} & $\mathrm{CE}$ & $238 \mathrm{~K}$ & $95.80 \%$ & - \\
\hline & $\mathrm{CE}$ & $100 \mathrm{~K}$ & $96.84 \%$ & - \\
\hline & $\mathrm{CE}$ & $886 \mathrm{~K}$ & $\mathbf{9 7 . 2 2} \%$ & - \\
\hline & $\mathrm{CE}$ & & $95.71 \%$ & $95.35 \%$ \\
\hline \multirow[t]{2}{*}{ LG-Net3 (ours) } & $\mathrm{CE}+\mathrm{ST}$ & $74 \mathrm{~K}$ & $96.46 \%$ & $95.39 \%$ \\
\hline & $\mathrm{CE}+\mathrm{TT}$ & & $96.82 \%$ & $95.77 \%$ \\
\hline \multirow{3}{*}{ LG-Net6 (ours) } & $\ddot{\mathrm{CE}}$ & & $97.03 \%$ & $96.45 \%$ \\
\hline & $\mathrm{CE}+\mathrm{ST}$ & $313 \mathrm{~K}$ & $97.13 \%$ & $96.57 \%$ \\
\hline & $\mathrm{CE}+\mathrm{TT}$ & & $97.67 \%$ & $96.79 \%$ \\
\hline
\end{tabular}

* represents the direct result of the corresponding paper.

Table 3: Comparison of FRR (false reject rate) of TC-ResNet 141.5 and $L G-N e t 6, F R R$ (false alarm rate) is at $0.5 \%$

\begin{tabular}{lccc}
\hline Model & Params & Loss & FRR \\
\hline \multirow{3}{*}{ TC-ResNet14-1.5 } & \multirow{2}{*}{$313 \mathrm{~K}$} & CE & $5.91 \%$ \\
& & CE+ST & $5.36 \%$ \\
& & CE & $5.29 \%$ \\
\hline \multirow{3}{*}{ LG-Net6 (ours) } & \multirow{2}{*}{$313 \mathrm{~K}$} & CE+ST & $4.69 \%$ \\
& & CE+TT & $\mathbf{3 . 5 6} \%$ \\
\hline
\end{tabular}

\subsection{Experimental Results and Analysis}

Impact of text anchor based metric learning method. As Table 2 shows, for each model, training with $C E+T T$ loss shows consistent improvements over training with $C E+S T$ loss, since that BERT provides informative and robust text anchors, which alleviates the effects of the acoustic environment and speakers. Furthermore, We find that training with $C E+S T$ loss yields a performance gain compared to CE loss because triplet loss increases the distinguishability of features. From Table 3, we can see that LG-Net6 achieves a lower FRR at $0.5 \%$ FAR. Note that there is no increase in memory footprint and computational resource at inference compared with the corresponding baseline. As Figure 4 shows, we randomly sample speech embeddings and use the t-SNE algorithm [23] to visualize the embeddings. We can see that keywords with similar pronunciation are difficult to distinguish (e.g. "go" and "no"), but are easily distinguished in the feature space after using our proposed text anchor based metric learning method.

Impact of other text vectors. We make a step forward to

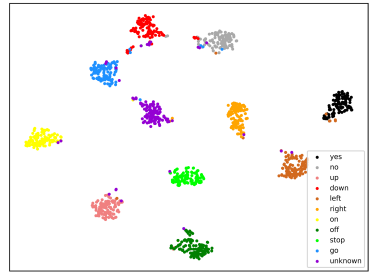

(a) Trained by CE loss

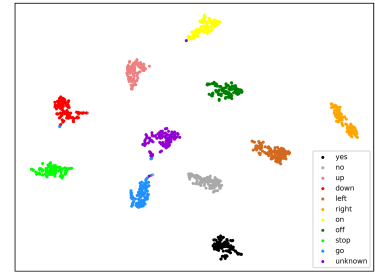

(b) Trained by proposed text based triplet los
Figure 4: $t$-SNE visualization of speech embeddings extracted by $L G$-Net6.

Table 4: Comparison of other text embeddings on GSCDv2

\begin{tabular}{lcc}
\hline Model & Text Embedding & Acc. \\
\hline & glove.840B.300d [21] & $96.21 \%$ \\
TC-ResNet14-1.5 [13] & BERT-Layer 1 [17] & $\mathbf{9 6 . 2 7 \%}$ \\
(Choi et al. 2019) & BERT-Layer 2 [17] & $96.23 \%$ \\
& BERT-Layer 12 [17] & $96.22 \%$ \\
\hline & glove.840B.300d [21] & $96.47 \%$ \\
LG-Net6 (ours) & BERT-Layer 1 [17] & $\mathbf{9 6 . 7 9 \%}$ \\
& BERT-Layer 2 [17] & $96.52 \%$ \\
& BERT-Layer 12 [17] & $96.50 \%$ \\
\hline
\end{tabular}

study how other text vectors influence the performance of our proposed method. Comparison experiments among the different layers of BERT and Glove [21] are performed. Referring to Table 4, both models, TC-ResNet14-1.5 [13] and LG-Net6, achieve the best performances by employing the output of the first layer from BERT as the text anchor. The results indicate that the lower layer of BERT can mostly capture word-level information, which is consistent with the statement in [22].

Impact of the local-global block. As shown in Table 2, compared to models trained using CE loss, LG-Nets achieve a balance between model size and accuracy. Compared to TCResNet14-1.5 [13], our LG-Net6 achieves better accuracy of 97.03\% and $96.45 \%$ on GSCDv1 and GSCDv2, respectively. Compared to NAS2 [9], our proposed LG-Net6 achieves comparable results using only $35 \%(313 \mathrm{~K} / 886 \mathrm{~K})$ of the number of parameters. The small-footprint model LG-Net3 obtains 95.71\% KWS accuracy in GSCDv1 and $95.35 \%$ KWS accuracy in GSCDv2. Visualization results (Figure 1b and Figure 1c) show that the self-attention layer helps the network capture long-term temporal information while preserving the short-term temporal information captured by the 1D-CNN.

\section{Conclusion}

In this paper, we propose text anchor based metric learning method and design a neural network LG-Net for small-footprint keyword spotting (KWS). The experimental results show that the proposed text anchor based metric learning method shows consistent improvements over the speech anchor based method. Moreover, our LG-Net model achieves SOTA accuracy on the Google Speech Commands Dataset version 1 and version 2.

\section{Acknowledgements}

This paper was partially supported by Shenzhen Science \& Technology Fundamental Research Programs (No: JSGG20191129105421211 \& GXWD2020123116580700720200814115301001) 


\section{References}

[1] J. Huh, M. Lee, H. Heo, S. Mun, and J. S. Chung, "Metric learning for keyword spotting," arXiv preprint arXiv:2005.08776, 2020.

[2] A. Raju, S. Panchapagesan, X. Liu, A. Mandal, and N. Strom, "Data augmentation for robust keyword spotting under playback interference," arXiv preprint arXiv:1808.00563, 2018.

[3] B. Liu, S. Nie, Y. Zhang, S. Liang, Z. Yang, and W. Liu, "Loss and double-edge-triggered detector for robust small-footprint keyword spotting," in ICASSP 2019-2019 IEEE International Conference on Acoustics, Speech and Signal Processing (ICASSP). IEEE, 2019, pp. 6361-6365.

[4] K. Zhang, Z. Wu, D. Yuan, J. Luan, J. Jia, H. Meng, and B. Song, "Re-weighted interval loss for handling data imbalance problem of end-to-end keyword spotting," Proc. Interspeech 2020, pp. 2567-2571, 2020.

[5] R. Prabhavalkar, R. Alvarez, C. Parada, P. Nakkiran, and T. N. Sainath, "Automatic gain control and multi-style training for robust small-footprint keyword spotting with deep neural networks," in 2015 IEEE International Conference on Acoustics, Speech and Signal Processing (ICASSP). IEEE, 2015, pp. 4704-4708.

[6] J. Hou, Y. Shi, M. Ostendorf, M.-Y. Hwang, and L. Xie, "Mining effective negative training samples for keyword spotting," in ICASSP 2020-2020 IEEE International Conference on Acoustics, Speech and Signal Processing (ICASSP). IEEE, 2020, pp. 7444 7448.

[7] N. Sacchi, A. Nanchen, M. Jaggi, and M. Cernak, "Openvocabulary keyword spotting with audio and text embeddings," in INTERSPEECH 2019-IEEE International Conference on Acoustics, Speech, and Signal Processing, no. CONF, 2019.

[8] G. Chen, C. Parada, and G. Heigold, "Small-footprint keyword spotting using deep neural networks," in 2014 IEEE International Conference on Acoustics, Speech and Signal Processing (ICASSP). IEEE, 2014, pp. 4087-4091.

[9] T. N. Sainath and C. Parada, "Convolutional neural networks for small-footprint keyword spotting," in Sixteenth Annual Conference of the International Speech Communication Association, 2015.

[10] R. Tang and J. Lin, "Deep residual learning for small-footprint keyword spotting," in 2018 IEEE International Conference on Acoustics, Speech and Signal Processing (ICASSP). IEEE, 2018 , pp. 5484-5488.

[11] C. Shan, J. Zhang, Y. Wang, and L. Xie, "Attention-based end-toend models for small-footprint keyword spotting," arXiv preprint arXiv:1803.10916, 2018.

[12] H. Zhang, J. Zhang, and Y. Wang, "Sequence-to-sequence models for small-footprint keyword spotting," arXiv preprint arXiv:1811.00348, 2018.

[13] S. Choi, S. Seo, B. Shin, H. Byun, M. Kersner, B. Kim, D. Kim, and S. Ha, "Temporal convolution for real-time keyword spotting on mobile devices," arXiv preprint arXiv:1904.03814, 2019.

[14] Y. Bai, J. Yi, J. Tao, Z. Wen, Z. Tian, C. Zhao, and C. Fan, "A time delay neural network with shared weight self-attention for smallfootprint keyword spotting," Proc. Interspeech 2019, pp. 2190 2194, 2019.

[15] X. Li, X. Wei, and X. Qin, "Small-footprint keyword spotting with multi-scale temporal convolution," arXiv preprint arXiv:2010.09960, 2020.

[16] T. Mo, Y. Yu, M. Salameh, D. Niu, and S. Jui, "Neural architecture search for keyword spotting," arXiv preprint arXiv:2009.00165, 2020.

[17] J. Devlin, M.-W. Chang, K. Lee, and K. Toutanova, "Bert: Pretraining of deep bidirectional transformers for language understanding," arXiv preprint arXiv:1810.04805, 2018.

[18] A. Vaswani, N. Shazeer, N. Parmar, J. Uszkoreit, L. Jones, A. N. Gomez, Ł. Kaiser, and I. Polosukhin, "Attention is all you need," in Advances in neural information processing systems, 2017, pp. 5998-6008
[19] P. Warden, "Speech commands: A dataset for limited-vocabulary speech recognition," arXiv preprint arXiv:1804.03209, 2018.

[20] A. Paszke, S. Gross, S. Chintala, G. Chanan, E. Yang, Z. DeVito, Z. Lin, A. Desmaison, L. Antiga, and A. Lerer, "Automatic differentiation in pytorch," 2017.

[21] J. Pennington, R. Socher, and C. D. Manning, "Glove: Global vectors for word representation," in Proceedings of the 2014 conference on empirical methods in natural language processing (EMNLP), 2014, pp. 1532-1543.

[22] G. Jawahar, B. Sagot, and D. Seddah, "What does bert learn about the structure of language?" in ACL 2019-57th Annual Meeting of the Association for Computational Linguistics, 2019 


\title{
Paper Template for INTERSPEECH 2021
}

\author{
Author Name ${ }^{1}$, Co-author Name ${ }^{2}$ \\ ${ }^{1}$ Author Affiliation \\ ${ }^{2}$ Co-author Affiliation \\ author@university.edu, coauthor@company.com
}

\begin{abstract}
For your paper to be published in the conference proceedings, you must use this document as both an instruction set and as a template into which you can type your own text. If your paper does not conform to the required format, you will be asked to fix it.

Please do not reuse your past papers as a template. To prepare your paper for submission, please always download a fresh copy of this template from the conference website and please read the format instructions in this template before you use it for your paper.

Conversion to PDF may cause problems in the resulting PDF or expose problems in your source document. Before submitting your final paper in PDF, check that the format in your paper PDF conforms to this template. Specifically, check the appearance of the title and author block, the appearance of section headings, document margins, column width, column spacing, and other features such as figure numbers, table numbers and equation number. In summary, you must proofread your final paper in PDF before submission.

The maximum number of pages is 5 . The $5^{\text {th }}$ page is reserved for references, exclusively. However, the references may begin on an earlier page immediately after the Acknowledgements section, and continue onto the $5^{\text {th }}$ page. If no space is available on an earlier page, then the references may begin on the $5^{\text {th }}$ page.

Index terms should be included as shown below.

Index Terms: speech recognition, human-computer interaction, computational paralinguistics
\end{abstract}

\section{Introduction}

This template can be found on the conference website. Templates are provided for Microsoft Word $\AA$, and $\mathrm{LT}_{\mathrm{E}} \mathrm{X}$. However, we highly recommend using $\mathrm{LT}_{\mathrm{E}} \mathrm{X}$ when preparing your submission. Information for full paper submission is available on the conference website.

\section{Page layout and style}

Authors should observe the following rules for page layout. A highly recommended way to meet these requirements is to use a given template (Microsoft Word ${ }^{\circledR}$ or $\mathrm{LT}_{\mathrm{E}} \mathrm{X}$ ) and check details against the corresponding example PDF file. Given templates, Microsoft Word ${ }^{\circledR}$ or LTEX, can be adapted/imported easily in other software such as LibreOffice, Apple Pages, LuaLTEX, and $\mathrm{XeLT}_{\mathrm{E}} \mathrm{X}$, but please be careful to match the layout of the provided PDF example.

\subsection{Basic layout features}

- Proceedings will be printed in DIN A4 format. Authors must submit their papers in DIN A4 format.
- Two columns are used except for the title section and for large figures that may need a full page width.

- Left and right margin are $20 \mathrm{~mm}$ each.

- Column width is $80 \mathrm{~mm}$.

- Spacing between columns is $10 \mathrm{~mm}$.

- Top margin is $25 \mathrm{~mm}$ (except for the first page which is $30 \mathrm{~mm}$ to the title top).

- Bottom margin is $35 \mathrm{~mm}$.

- Text height (without headers and footers) is maximum $235 \mathrm{~mm}$.

- Headers and footers must be left empty.

- Check indentations and spacings by comparing to this example file (in PDF).

\subsubsection{Headings}

Section headings are centered in boldface with the first word capitalized and the rest of the heading in lower case. Sub- headings appear like major headings, except they start at the left margin in the column. Sub-sub-headings appear like sub-headings, except they are in italics and not boldface. See the examples in this file. No more than 3 levels of headings should be used.

\subsection{Text font}

Times or Times Roman font is used for the main text. Font size in the main text must be 9 points, and in the References section 8 points. Other font types may be used if needed for special purposes. It is VERY IMPORTANT that while making the final PDF file, you embed all used fonts! To embed the fonts, you may use the following instructions:

1. For Windows users, the bullzip printer can convert any PDF to have embedded and subsetted fonts.

2. For Linux/Mac users, you may use pdftops file.pdf pstopdf -dPDFSETTINGS=/prepress file.pdf

LTEXusers: users should use Adobe Type 1 fonts such as Times or Times Roman. These are used automatically by the INTERSPEECH2021.sty style file. Authors must not use Type 3 (bitmap) fonts.

\subsection{Figures}

All figures must be centered on the column (or page, if the figure spans both columns). Figure captions should follow each figure and have the format given in Figure 1.

Figures should be preferably line drawings. If they contain gray levels or colors, they should be checked to print well on a high-quality non-color laser printer. 


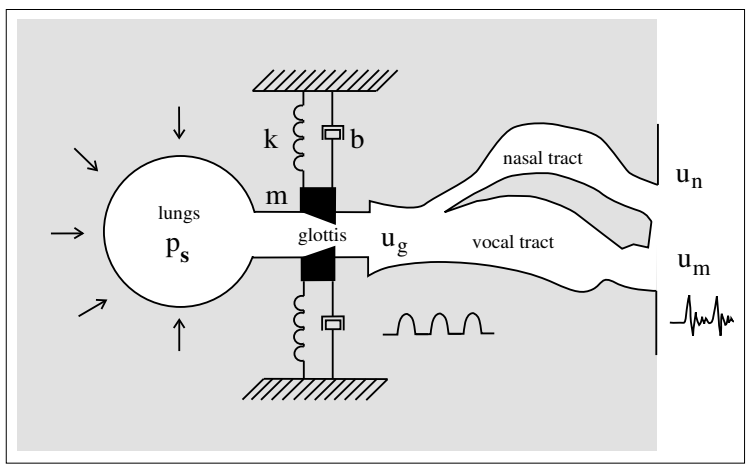

Figure 1: Schematic diagram of speech production.

Graphics (i.e., illustrations, figures) must not use stipple fill patterns because they will not reproduce properly in Adobe PDF. Please use only SOLID FILL COLORS.

Figures which span 2 columns (i.e., occupy full page width) must be placed at the top or bottom of the page.

\subsection{Tables}

An example of a table is shown in Table 1. The caption text must be above the table.

Table 1: This is an example of a table

\begin{tabular}{cc}
\hline \multicolumn{1}{c}{ Ratio } & Decibels \\
\hline $1 / 10$ & -20 \\
$1 / 1$ & 0 \\
$2 / 1$ & $\approx 6$ \\
$3.16 / 1$ & 10 \\
$10 / 1$ & 20 \\
$100 / 1$ & 40 \\
$1000 / 1$ & 60 \\
\hline
\end{tabular}

\subsection{Equations}

Equations should be placed on separate lines and numbered. Examples of equations are given below. Particularly,

$$
x(t)=s\left(f_{\omega}(t)\right)
$$

where $f_{\omega}(t)$ is a special warping function

$$
f_{\omega}(t)=\frac{1}{2 \pi j} \oint_{C} \frac{\nu^{-1 k} \mathrm{~d} \nu}{\left(1-\beta \nu^{-1}\right)\left(\nu^{-1}-\beta\right)}
$$

A residue theorem states that

$$
\oint_{C} F(z) \mathrm{d} z=2 \pi j \sum_{k} \operatorname{Res}\left[F(z), p_{k}\right]
$$

Applying (3) to (1), it is straightforward to see that

$$
1+1=\pi
$$

Finally we have proven the secret theorem of all speech sciences. No more math is needed to show how useful the result is!
Table 2: Main predefined styles in Word

\begin{tabular}{ll}
\hline Style Name & Entities in a Paper \\
\hline Title & Title \\
Author & Author name \\
Affiliation & Author affiliation \\
Email & Email address \\
AbstractHeading & Abstract section heading \\
Body Text & First paragraph in abstract \\
Body Text Next & Following paragraphs in abstract \\
Index & Index terms \\
1. Heading 1 & $1^{\text {st level section heading }}$ \\
1.1 Heading 2 & $2^{\text {nd }}$ level section heading \\
1.1.1 Heading 3 & $3^{\text {rd level section heading }}$ \\
Body Text & First paragraph in section \\
Body Text Next & Following paragraphs in section \\
Figure Caption & Figure caption \\
Table Caption & Table caption \\
Equation & Equations \\
• List Bullet & Bulleted lists \\
[1] Reference & References \\
\hline
\end{tabular}

\subsection{Information for Word users only}

For ease of formatting, please use the styles listed in Table 2. The styles are defined in this template file and are shown in the order in which they would be used when writing a paper. When the heading styles in Table 2 are used, section numbers are no longer required to be typed in because they will be automatically numbered by Word. Similarly, reference items will be automatically numbered by Word when the "Reference" style is used.

If your Word document contains equations, you must not save your Word document from ".docx" to ".doc" because when doing so, Word will convert all equations to images of unacceptably low resolution.

\subsection{Hyperlinks}

For technical reasons, the proceedings editor will strip all active links from the papers during processing. Hyperlinks can be included in your paper, if written in full, e.g. "http://www.foo.com/index.html". The link text must be all black. Please make sure that they present no problems in printing to paper.

\subsection{Multimedia files}

The INTERSPEECH organizing committee offers the possibility to submit multimedia files. These files are meant for audiovisual illustrations that cannot be conveyed in text, tables and graphs. Just like you would when including graphics, make sure that you have sufficient author rights to the multimedia materials that you submit for publication. The proceedings media will NOT contain readers or players, so be sure to use widely accepted file formats, such as MPEG, Windows WAVE PCM (.wav) or Windows Media Video (.wmv) using standard codecs.

Your multimedia files must be submitted in a single ZIP file for each separate paper. Within the ZIP file you can use folders and filenames to help organize the multimedia files. In the ZIP file you should include a TEXT or HTML index file which describes the purpose and significance of each multimedia file. From within the manuscript, refer to a multimedia illustration 
by its filename. Use short file names without blanks for clarity.

The ZIP file you submit will be included as-is in the proceedings media and will be linked to your paper in the navigation interface of the proceedings. Causal Productions (the publisher) and the conference committee will not check or change the contents of your ZIP file.

Users of the proceedings who wish to access your multimedia files will click the link to the ZIP file which will then be opened by the operating system of their computer. Access to the contents of the ZIP file will be governed entirely by the operating system of the user's computer.

\subsection{Page numbering}

Final page numbers will be added later to the document electronically. Do not make any footers or headers!

\subsection{References}

The reference format is the standard IEEE one. References should be numbered in order of appearance, for example [?], [?], [?, pp. 417-422], and [?].

\subsection{Abstract}

The total length of the abstract is limited to 200 words. The abstract included in your paper and the one you enter during webbased submission must be identical. Avoid non-ASCII characters or symbols as they may not display correctly in the abstract book.

\subsection{Author affiliation}

Please list country names as part of the affiliation for each country.

\subsection{Number of authors in the author list}

The maximum number of authors in the author list is twenty. If the number of contributing authors is more than twenty, they should be listed in a footnote or in acknowledgement section, as appropriate.

\subsection{Submitted files}

Authors are requested to submit PDF files of their manuscripts. You can use commercially available tools or for instance http://www.pdfforge.org/products/pdfcreator. The PDF file should comply with the following requirements: (a) there must be no PASSWORD protection on the PDF file at all; (b) all fonts must be embedded; and (c) the file must be text searchable (do CTRL-F and try to find a common word such as "the"). The proceedings editors (Causal Productions) will contact authors of non-complying files to obtain a replacement. In order not to endanger the preparation of the proceedings, papers for which a replacement is not provided in a timely manner will be withdrawn.

\section{Discussion}

This is the discussion. This is the discussion. This is the discussion. Is there any discussion?

Lorem ipsum dolor sit amet, consectetur adipiscing elit. Cras consequat mollis odio, nec venenatis enim auctor sed. Integer tincidunt fringilla lectus eget condimentum. In eget sapien id eros dapibus interdum vel ac quam. Aenean vitae rutrum erat.
Aenean et risus pharetra, lacinia augue ut, fermentum ante. Integer dui arcu, interdum at ornare a, faucibus quis est. Mauris quis quam felis. Etiam pulvinar massa et turpis lacinia, eu posuere mi iaculis. Fusce at velit quis leo dignissim porttitor.

Fusce ut nunc eu sapien venenatis finibus a vel ligula. Pellentesque habitant morbi tristique senectus et netus et malesuada fames ac turpis egestas. Ut quam eros, volutpat at gravida consectetur, rutrum ut leo. Aenean cursus euismod feugiat. Cras hendrerit, ligula eu feugiat malesuada, neque turpis auctor lacus, sit amet accumsan neque orci a quam. Mauris suscipit ultrices mattis. Nulla at interdum metus, id pharetra diam. Curabitur at vestibulum sem, sed elementum massa. Donec iaculis et arcu ut rutrum. Fusce gravida, mauris porta volutpat eleifend, enim mauris eleifend orci, eu ultrices leo purus vitae metus. In pretium dolor ut magna dictum, at imperdiet lectus porta.

Quisque mollis lectus id risus pretium mattis. Morbi scelerisque posuere est, id efficitur urna luctus non. Praesent quam lacus, facilisis id ante eu, vehicula maximus ex. Nullam mollis in arcu vitae efficitur. Aliquam molestie eleifend ante, in pretium velit ultrices ac. Etiam laoreet nec sem non pulvinar. Integer ligula felis, interdum non lacus id, malesuada imperdiet turpis.

Aenean sit amet volutpat nisi. Aliquam eu erat quis tortor ultrices laoreet. Vivamus fermentum semper metus, non faucibus libero euismod vitae. Sed efficitur porta congue. Aenean in faucibus nisi. Donec suscipit augue vitae orci consequat, sit amet aliquet felis varius. Duis efficitur lacinia dolor sit amet lobortis. Curabitur erat sapien, molestie nec nisi eu, dignissim accumsan ipsum. Fusce id nibh nec risus dictum posuere in ac magna. Donec malesuada massa sed erat lacinia cursus. Suspendisse ornare augue nec volutpat consequat.

Vestibulum et vulputate nisi, a malesuada mi. Nam pellentesque arcu sapien, at placerat odio imperdiet ut. Curabitur nec venenatis tellus, vel aliquet nisi. Curabitur vel ligula sit amet metus auctor pretium. Nullam nulla mi, blandit a mattis id, vulputate sit amet enim. Proin mollis fringilla dictum. Proin lacinia orci purus.

Curabitur porttitor bibendum dolor, nec consectetur sapien pulvinar id. Donec eleifend, est vel dignissim pretium, tortor augue euismod nunc, id fermentum erat felis ac neque. Morbi id lectus ultricies, rutrum justo eu, sollicitudin risus. Suspendisse lobortis efficitur nisi sit amet pellentesque. Ut eget augue at mi aliquet mattis. Proin et feugiat erat, sit amet sodales eros. Integer sed elit quis est mattis ullamcorper. Pellentesque lectus nisi, vulputate a imperdiet tincidunt, auctor nec orci. Pellentesque sagittis nisl orci, vitae placerat massa lacinia nec. Sed egestas magna sed augue sollicitudin luctus. Praesent interdum bibendum tortor, eu porta purus. Aliquam convallis velit id mi fermentum, sed ornare eros cursus.

Quisque congue leo a fringilla pharetra. Phasellus sed tempor est, sed auctor purus. Morbi vel lacus ullamcorper, auctor mauris id, pulvinar lorem. Suspendisse potenti. Nam porta, purus non eleifend bibendum, erat metus pellentesque elit, non luctus nibh nunc ornare nisl. Sed rutrum lacinia nisi ac suscipit. Curabitur non blandit augue. Integer viverra, ipsum vel molestie euismod, sem quam tempus massa, eget efficitur ante turpis non metus. Quisque efficitur posuere velit in iaculis. Cras imperdiet varius urna vitae vestibulum. Donec accumsan eget nisi sed pellentesque. Vestibulum id quam ut urna volutpat ullamcorper gravida sit amet libero. Aliquam bibendum, ligula vitae porta malesuada, arcu diam congue erat, a pharetra diam sem vulputate tortor. Etiam luctus iaculis leo cursus tristique.

Mauris mattis sem dolor, sit amet ullamcorper arcu tincidunt ac. Vestibulum at blandit tortor. Quisque bibendum 
congue leo, vitae eleifend massa. Vestibulum vitae odio elit. Lorem ipsum dolor sit amet, consectetur adipiscing elit. Ut sagittis quam vel felis ornare, in gravida felis tempor. Donec molestie dui quis leo venenatis blandit. Nunc sit amet finibus metus. Cras ut lectus ex.

Suspendisse commodo libero vel leo tincidunt, a tempus mauris porta. Integer varius eros ac sapien lacinia vehicula. Donec porttitor, lacus faucibus rhoncus venenatis, neque quam imperdiet nunc, id consectetur metus purus quis sapien. Phasellus interdum nulla vel euismod posuere. Vestibulum finibus magna vel finibus mollis. Curabitur mollis turpis tortor, hendrerit vulputate justo egestas quis. Nam dignissim luctus leo non elementum. Phasellus a metus at leo malesuada bibendum. Mauris quis eleifend magna, nec vehicula ex. Donec venenatis urna fermentum commodo vehicula. Ut mattis scelerisque aliquam. Vivamus pulvinar erat metus, id tempus mi vulputate quis.

Fusce lobortis a urna eget blandit. Vivamus in eleifend neque, at sollicitudin lectus. Quisque faucibus egestas lorem, in commodo diam maximus eu. Morbi finibus ante ac felis porttitor euismod. Donec lobortis aliquam ipsum sit amet luctus. Cum sociis natoque penatibus et magnis dis parturient montes, nascetur ridiculus mus. Etiam rutrum neque sapien, eget luctus turpis iaculis pulvinar. Duis quis pulvinar nunc, nec bibendum ligula. Phasellus suscipit sagittis lacus molestie laoreet. Pellentesque lacus diam, tincidunt a aliquam vitae, aliquet non justo.

Etiam lectus lacus, commodo eget consectetur eget, auctor vitae leo. Praesent vitae erat in diam blandit semper vitae et eros. Maecenas auctor pharetra nibh eget egestas. Donec accumsan ut risus eget rhoncus. Nam placerat, erat sit amet gravida mollis, purus arcu accumsan diam, tempus pharetra risus mi ac sapien. Ut et tortor porta, pulvinar elit vitae, tempor mi. Nam interdum, nisl non pharetra molestie, turpis neque commodo ligula, sit amet pretium nisl nibh quis ante. Quisque et ex eget velit lobortis suscipit. Integer aliquam finibus molestie. Sed pellentesque neque eu turpis aliquet, mattis ornare enim finibus. In hac habitasse platea dictumst.

Integer congue quis justo a posuere. Quisque porta, ante et dignissim suscipit, arcu mauris ultrices libero, nec sollicitudin purus lacus a enim. Aliquam feugiat eget lacus molestie sodales. Duis blandit placerat nunc, et venenatis turpis dictum vel. Nulla facilisi. Nam ullamcorper, tellus eu posuere mattis, arcu lacus dictum nulla, vel mattis nisi sem posuere tellus. Etiam quis eros condimentum lectus lobortis eleifend. In ex lacus, sodales scelerisque egestas ac, aliquam nec purus. Nunc sit amet magna non libero ullamcorper dictum. Phasellus porta faucibus tempus. Praesent blandit tortor sed tellus ornare consectetur. Sed sed nisi id neque porta varius eu eu velit. Curabitur varius convallis justo id facilisis. Mauris auctor velit nec aliquam cursus.

Integer suscipit scelerisque leo sed faucibus. Ut commodo nulla luctus diam posuere egestas. Integer ut augue ac velit ullamcorper tempus. Pellentesque in mi rhoncus, sodales sem quis, commodo sem. Aenean dapibus euismod diam id rhoncus. Nullam vehicula placerat eros consectetur luctus. Aliquam auctor ipsum vitae egestas imperdiet. Ut nulla lacus, imperdiet quis urna vel, ornare imperdiet tortor. Mauris nec diam ac nunc laoreet volutpat at id turpis. Nulla eu neque a risus feugiat iaculis ac vel risus. Ut tempus elementum lorem eget porta. Nullam et ullamcorper urna. Cum sociis natoque penatibus et magnis dis parturient montes, nascetur ridiculus mus. Phasellus eget dui vitae nulla hendrerit ultrices quis rutrum leo.

Proin consectetur lacus sit amet eleifend varius. Etiam eu blandit risus. Curabitur pellentesque urna sed dolor congue mattis. Vestibulum ut velit posuere, feugiat leo a, dignissim massa. Proin eu nulla risus. Fusce luctus bibendum est, sit amet venenatis nisi finibus non. Donec ultricies ornare nunc at lobortis. Nam a auctor metus. Vestibulum pretium condimentum turpis ac mattis. Curabitur semper sagittis rhoncus. Duis molestie facilisis mattis. Sed pharetra lorem id tortor efficitur, sed maximus leo posuere. Quisque suscipit molestie convallis. Duis imperdiet placerat congue. Morbi placerat, velit ut tempor porta, ex nisi imperdiet purus, non feugiat ex velit nec nulla.

Phasellus mattis at erat eget lobortis. Vivamus sodales odio non erat luctus faucibus. Curabitur aliquam luctus nulla quis consectetur. Fusce vulputate finibus vulputate. Cras at condimentum massa. Duis vestibulum ipsum ac tortor lobortis fringilla. Cras non neque at nunc pellentesque mollis. Sed quis erat mauris. Ut dapibus sem lectus, quis imperdiet diam bibendum et. Maecenas quis venenatis ante. Nunc blandit a risus sed scelerisque. Praesent cursus est sit amet nisi tempus, quis placerat libero rutrum. Phasellus lacinia nisi quis consequat mollis. Phasellus sagittis aliquam lacus.

Sed dolor quam, posuere nec nunc eget, feugiat lobortis ligula. Fusce lacinia fermentum dolor, luctus dapibus ex venenatis feugiat. In hac habitasse platea dictumst. Nullam vitae ligula dignissim, interdum turpis quis, tincidunt metus. Nullam in nisl vitae mauris egestas porta. Nam fringilla aliquet sapien, non dapibus nunc sollicitudin id. Nunc hendrerit felis et vehicula consequat. Praesent varius libero id volutpat iaculis. Aliquam vel dui imperdiet, pharetra augue sed, iaculis nulla. Quisque mollis orci nec odio eleifend, eget laoreet nunc feugiat. Cras varius tortor a fringilla gravida.

Sed posuere erat eu dolor consequat euismod. Donec imperdiet, tellus nec convallis commodo, lorem sem lobortis purus, a lacinia massa ipsum vitae nisl. Vivamus auctor tellus in urna iaculis luctus. In dui nibh, posuere a erat a, lobortis finibus nulla. Sed vel suscipit nisi. Nunc eget nibh risus. Sed posuere tempus eleifend. Nullam ac lacinia ligula, ut blandit erat. In at est sed turpis consequat rutrum. Nunc eget lectus venenatis, convallis ligula non, mollis orci. Aliquam sit amet ligula turpis. Sed finibus laoreet elit nec molestie.

Mauris in nisi et neque euismod aliquam ut eget felis. Sed eget dictum tellus, finibus rutrum nibh. Fusce placerat augue a faucibus semper. Nam sed nisl ligula. Vivamus ante augue, faucibus at risus ut, hendrerit viverra risus. Etiam justo eros, dignissim a nunc sed, porta mollis erat. Fusce in sem accumsan, laoreet nibh porta, porttitor orci. Donec venenatis vehicula ante eget dictum.

Aliquam fermentum a metus pellentesque cursus. Vivamus eleifend ultricies tellus, vel scelerisque diam. Sed hendrerit est at elit suscipit placerat. Praesent nec pretium erat. Quisque blandit nunc in felis bibendum consequat.

\section{Conclusions}

Authors must proofread their PDF file prior to submission to ensure it is correct. Authors should not rely on proofreading the Word file. Please proofread the PDF file before it is submitted.

\section{Acknowledgements}

The ISCA Board would like to thank the organizing committees of the past INTERSPEECH conferences for their help and for kindly providing the template files.

Note to authors: Authors should not use logos in the acknowledgement section; rather authors should acknowledge corporations by naming them only. 